\title{
RETORNANDO AL HOTEL DE HILBERT
}

\author{
Juan Pablo Jorge y Hernán Vázquez
}

\begin{abstract}
RESUMEN. Se construyen particiones particulares del conjunto de los naturales a través de procesos recursivos generando, de esta manera, numerables ejemplos de conjuntos numerables y disjuntos cuya unión es un conjunto también numerable. El proceso es constructivo por lo cual no se hace uso del axioma de elección. Se presenta un programa que genera una de estas particiones especiales y se muestra cómo generar infinitas de las mismas. Esta línea de razonamiento puede tener múltiples aplicaciones en la teoría de conjuntos y de modelos. Probamos que la cantidad de formas de realizar estas particiones de los naturales es no numerable, existe mayor cantidad de estas particiones, bautizadas doblemente numerables, que números naturales. Para cada número natural mayor que 1, mostramos un procedimiento efectivo que genera estas particiones.
\end{abstract}

\begin{abstract}
Aвstract. Some partitions of Natural Number set are built through recursive processes generating in this manner countable examples of countable and disjoint sets whose union is a set also countable. This process is constructive, so the Axiom of choice is not used. We provide a PC program that generates one of these special partitions and shows how to generate infinite of them. This line of reasoning can have multiple applications in Set theory and Model theory. We proved that the number of ways to make these partitions of natural numbers is not countable, there are more of these partitions (named doubly countable) than natural numbers. For each natural number greater than 1, we show an effective procedure that generates these partitions.
\end{abstract}

\section{§1. Introducción}

La línea de razonamientos que desarrollaremos está basada en generar particiones de los números naturales (Vinogradov, 1977). Pero no cualquier partición de los mismos, como podría ser, por ejemplo, la que tiene sólo dos clases infinitas correspondientes a los pares e impares. Queremos particiones de los naturales en infinitas clases cada una numerable, además de un método efectivo para lograrlo. Separar a los números naturales en infinitos conjuntos numerables, disjuntos dos a dos, de forma recursiva es nuestra tarea principal. Llamaremos a estas particiones

Palabras clave: Particiones de los naturales; Hotel de Hilbert; Uniones numerables; Matemática aplicada.

Keywords:Partition of natural numbers; Hilbert's Hotel; Countable unions; Applied mathematics. 
de los naturales, particiones doblemente numerables (PDN), ya que todos sus conjuntos son numerables y tenemos una cantidad numerable de estos. A lo largo de todo el trabajo, entenderemos numerable como la cardinalidad de los números naturales.

Pensando en el famoso Hotel de Hilbert (El hotel de Hilbert, 2020; El hotel infinito de Hilbert, 2021; Paenza, 2005), nuestra primera tarea se podría definir de la siguiente manera: si llegaran simultáneamente a la isla donde se encuentra el hotel numerables contingentes de turistas y cada contingente fuese numerable, ¿cómo encontrarle una habitación diferente a cada turista si el hotel se encuentra en su totalidad ya ocupado?

Este problema está muy relacionado con el siguiente (problema de la unión numerable): dada una cantidad numerable de conjuntos disjuntos de a pares, tales que cada uno es numerable, ¿cuál es la cardinalidad del conjunto unión? Es sabido que en la axiomática de Zermelo-Fraenkel (ZF) (Ivorra, 2018a, 2018b; Suppes, 1968) es necesario contar con el Axioma de Elección (AE) si se quiere asegurar que la cardinalidad de la unión sea numerable para el caso general (Rubin y Rubin, 1963). Al generar las particiones de los naturales buscadas, nosotros encontraremos infinitos ejemplos de conjuntos numerables y disjuntos cuya unión es el conjunto de los números naturales. Es decir, generaremos con un procedimiento efectivo al menos numerables ejemplos de conjuntos numerables y disjuntos de a pares, tales que su unión es numerable. La diferencia principal es que nosotros estamos interesados en el proceso inverso, en vez de unir numerable conjuntos numerables (y disjuntos), generar particiones doblemente numerables de los naturales. Este proceso nos llevará a una prueba original del problema de la unión numerable nombrado.

Nuestros resultados principales están dentro del dominio de la matemática recursiva, lo resolveremos de manera algorítmica y mostraremos un ejemplo de programa posible que realiza una de las infinitas particiones posibles.

\section{§2. Conceptos matemáticos fundamentales}

Antes de comenzar a exponer el núcleo de nuestro trabajo, nos detendremos para explicitar algunos conceptos básicos que usaremos. Será esencial dominar los conceptos de equipotencia o coordinabilidad entre conjuntos, cardinalidad, partición y relación de equivalencia. Presentaremos, además, el problema del Hotel de Hilbert en sus versiones clásicas. Quienes tengan presente estos temas pueden pasar a la sección siguiente.

2.1. Biyección, equipotencia y Teorema de Cantor-Bernstein. Uno de los conceptos más importantes a la hora de realizar nuestra tarea es el de función biyectiva (ver definición (2.3)). La importancia de las funciones biyectivas radica en que nos permiten contar cantidad de elementos de los conjuntos aun cuando los conjuntos 
no son finitos. Y uno de nuestros objetivos es generar particiones cuyas clases tengan todas la misma cantidad de elementos. Cualquier persona que haya entrado alguna vez a una sala en donde todas sus butacas estuviesen ocupadas y ninguna persona quedara parada pudo deducir que existían tantas butacas como personas dentro de la sala (suponiendo que ninguna butaca tiene más de una persona). Esto es importante, ya que podemos saber si hay tantas butacas como personas sin necesidad de saber exactamente cuántas hay, esto es, sin necesidad de contar ni butacas ni personas. En este caso, estamos habilitados para decir que el conjunto de butacas es equipotente con el conjunto de personas dentro de la sala. Puede ser que no sepamos exactamente cuántas personas hay, pero estamos seguros de que existe la misma cantidad de asientos. Lo dicho hasta acá puede ser condensado en las siguientes definiciones:

Definición 2.1. Sean $X, Y$ conjuntos y $f$ una función, tal que $f: X \rightarrow Y$. Decimos que $f$ es inyectiva si

$$
(\forall x \in X)(\forall y \in X)(x \neq y \Rightarrow f(x) \neq f(y))
$$

Es decir, dados dos elementos cualesquiera distintos del dominio, sus imágenes no pueden coincidir. Si existe una función inyectiva $f: X \rightarrow Y$, estamos en condiciones de asegurar que la cantidad de elementos de $X$ es menor o igual que la cantidad de elementos de $Y$ (ver Figura 1). Si a la cantidad de elementos de un conjunto $X$ la llamamos cardinalidad de $X$, y la denotamos como $|X|$, entonces podemos resumir lo anterior diciendo que si existe una función inyectiva entre $X$ e $Y,|X| \leq|Y|$.

Definimos la sobreyectividad de una función $f$ como la propiedad que nos garantiza que todo elemento del conjunto de llegada o codominio $(Y)$ esté relacionado con algún elemento del dominio $(X)$.

Definición 2.2. Sean $X, Y$ conjuntos y $f$ una función, tal que $f: X \rightarrow Y$. Decimos que f es sobreyectiva si

$$
(\forall y \in Y) \quad(\exists x \in X) \quad \text { tal que } \quad f(x)=y .
$$

En el caso de existir una función sobreyectiva como la dada en la definición anterior, podemos asegurar que $|Y| \leq|X|$. Puede observarse en la Figura 2 esta relación necesaria entre la cantidad de elementos de los conjuntos.

Definición 2.3. Bajo las mismas condiciones de las definiciones anteriores, decimos que una función $f$ es biyectiva si es inyectiva y sobreyectiva. 


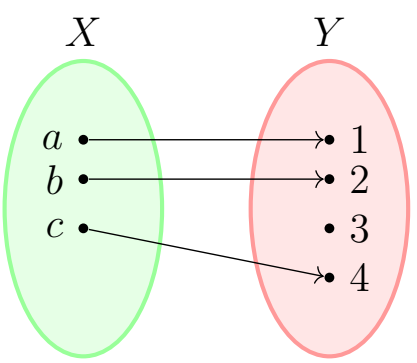

Figura 1: Función inyectiva

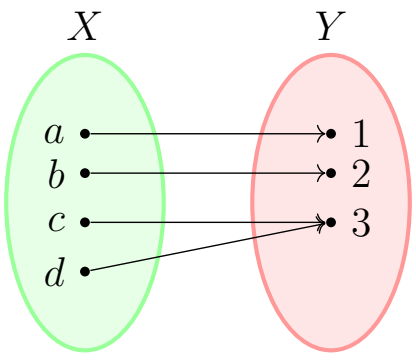

Figura 2: Función sobreyectiva

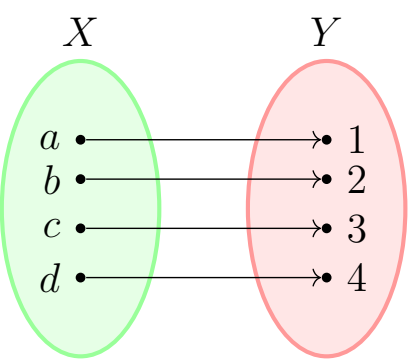

Figura 3: Función biyectiva

Estamos ahora en condiciones de definir uno de los conceptos centrales para el desarrollo de nuestro artículo, el concepto de equipotencia.

Definición 2.4. Sean $X$ e $Y$ dos conjuntos. Decimos que $X$ es equipotente con $Y$ si existe una función biyectiva $f$, tal que $f: X \rightarrow Y$.

La equipotencia entre conjuntos implica que los mismos comparten la cardinalidad (Figura 3). Cuando un conjunto $X$ es equipotente con el conjunto de los números naturales, es decir, existe $f: X \rightarrow \mathbb{N}$ biyectiva, decimos que el conjunto $X$ es numerable. Por supuesto, si existe esta función $f$ biyectiva, también existe su función inversa, $f^{-1}: \mathbb{N} \rightarrow X$. Lo último significa que la relación de ser equipotente es simétrica, ya que la inversa de toda función biyectiva es otra función biyectiva. Esto es, si $X$ es equipotente con $Y$, entonces $Y$ es equipotente con $X$ para cualesquiera $X$ e $Y$.

Como también sabemos, cada conjunto $X$ tiene al menos una función biyectiva que lo relaciona consigo mismo; la función identidad $\mathbb{I}: X \rightarrow X$, tal que $f(x)=x$ para todo elemento del dominio. Cuando una relación satisface que todo elemento de su dominio está relacionado consigo mismo, decimos que la relación es reflexiva. Por lo tanto, como todo conjunto es equipotente consigo mismo, ser equipotente es una relación reflexiva.

La última propiedad que destacaremos de la relación de equipotencia es su transitividad, es decir, si $X$ es equipotente con $Y$, y a su vez, $Y$ lo es con $Z$, entonces $X$ y $Z$ son equipotentes. Esto se debe a que la composición de funciones biyectivas es biyectiva. Si un conjunto $X$ es equipotente con el conjunto $\{1,2,3,4, \ldots, n\}$, decimos que su cardinalidad, la cantidad de elementos del mismo, es $n$. También decimos que es equipotente con una sección de longitud $n$ de los Naturales. Si $X$ es equipotente con el conjunto vacío, su cardinalidad es 0 . Cuando la cardinalidad de un conjunto $X$ es un número natural (o cero), el conjunto es finito. Puede probarse que el único conjunto equipotente con el conjunto vacío es él mismo, es decir, sólo existe un conjunto que no tiene ningún elemento (el conjunto vacío es único).

Nuestro interés en la equipotencia radica en su utilidad a la hora de comparar cardinalidades de conjuntos, es decir, comparar la cantidad de elementos que tienen los conjuntos. En la axiomática de conjuntos de ZFC, los números cardinales se definen a partir de otros conjuntos llamados ordinales (Ivorra, 2018a; Suppes, 1968). Para que esa definición sea aplicable a todos los conjuntos, es decir, para que todo conjunto tenga asignado un único cardinal, es necesario contar con el Axioma de Elección. Como la intención de este artículo es trabajar fuera del ámbito de este axioma, pero necesitamos comparar cardinales de conjuntos infinitos, presentaremos el Teorema de Cantor-Schröder-Bernstein (CSB). La 
historia detrás de este teorema (y cómo su demostración pudo independizarse del AE) es muy interesante. Para quienes deseen una introducción amena en el tema se recomienda (Basa, 2014).

Teorema 2.5 (Cantor-Schröder-Bernstein (CSB)). Sean $X$ e $Y$ conjuntos. Si existen funciones inyectivas $f$ y $g$, tales que $f: X \rightarrow Y$ y $g: Y \rightarrow X$, entonces existe una función biyectiva $h$, tal que $h: X \rightarrow Y$.

El Teorema CSB nos dice que la relación tener cardinal menor o igual que es una relación antisimétrica. Este resultado será crucial a la hora de probar el Teorema 5.1.

El objetivo de este artículo es desarrollar particiones particulares de los números naturales. Por lo tanto, debemos decir qué entendemos por una partición. Debido a que este concepto está íntimamente relacionado con el de relación de equivalencia, procederemos a dar las últimas tres definiciones de esta sección: relación de equivalencia, partición y Axioma de Elección.

\subsection{Particiones y relaciones de equivalencia.}

Definición 2.6. Se dice que una relación es de equivalencia si y sólo si es a la vez

- reflexiva: todo elemento del dominio se relaciona consigo mismo: $\forall x(x \mathcal{R} x)$;

- simétrica: si $x$ se relaciona con y, entonces y se relaciona con $x: \forall x \forall y(x \mathcal{R} y \Rightarrow y \mathcal{R} x)$;

- transitiva: si $x$ se relaciona con y e y se relaciona con $z$, entonces $x$ se relaciona con $z$ : $\forall x \forall y \forall z(x \mathcal{R} y \wedge y \mathcal{R} z \Rightarrow x \mathcal{R} z)$.

Por lo tanto, la relación de equipotencia entre conjuntos es una relación de equivalencia. Como ejemplo podemos nombrar que tanto los números pares como los impares son equipotentes con el conjunto de los números naturales. Esto se encuentra relacionado con el hecho de que existe una partición de los números naturales formada por los pares y los impares. Por otro lado, como los Naturales y los Enteros son equipotentes, tenemos que los pares o impares tienen la misma cantidad de elementos que los Enteros (usando la transitividad de la relación de equipotencia). Utilizando el concepto de biyección es como Cantor demostró que los números racionales $\mathbb{Q}$ son numerables, esto es, que $\mathbb{N}$ y $\mathbb{Q}$ son equipotentes (para más detalles (Apostol, 1984, 1998; Vinogradov, 1977)).

Cuando $X$ es equipotente con el conjunto de los números naturales ( $X$ numerable), se dice que su cardinal es Aleph subcero (Aleph se acentúa en la primera sílaba y la Real Academia Española acepta su grafía y fonética como álef) y se denota $\aleph_{0}$. Todos los conjuntos numerables tienen el mismo cardinal, $\aleph_{0}$. Cuando se cumpla lo anterior, escribiremos

$$
|X|=\aleph_{0} .
$$

Esto es central en nuestro trabajo, ya que las PDN cumplen la ecuación anterior, son todas equipotentes con los Naturales.

Las particiones tienen una vínculo muy estrecho con las relaciones de equivalencia: toda relación de equivalencia definida sobre elementos de un conjunto $X$ genera una única partición de este conjunto. ¿Qué queremos decir con una partición? Intuitivamente, 
una partición de un conjunto $X$ es una forma de generar subconjuntos de $X$, tales que sean disjuntos, es decir, su intersección sea vacía, y que además su unión sea el conjunto original $X$ (no puede quedarme ningún elemento fuera). Por ejemplo, el conjunto de los números pares y el de los impares forman una partición de los Naturales en dos subconjuntos. Los números positivos y los negativos no forman una partición del conjunto de los números enteros, ya que, a pesar de ser disjuntos, no contienen al cero. Si el 0 es incluido en alguno de los dos conjuntos, entonces tenemos una partición. Podríamos también agregar un conjunto que sólo tenga al 0, en este caso tendríamos una partición de los Enteros, pero ahora de 3 elementos (subconjuntos): positivos, negativos y el nuevo conjunto con el cero. No es necesario que los elementos de la partición tengan la misma cantidad de elementos. Nuestro objetivo es generar particiones de los Naturales donde todos sus elementos (subconjuntos de naturales) sean equipotentes.

Definición 2.7. Sea $\left\{X_{i}\right\}_{i \in \Delta}$ una familia de subconjuntos no vacíos de $X$, con i perteneciente a un conjunto de subindices $\Delta$ fijo. Decimos que esta familia es una partición de $X$ si

- $X=\bigcup_{i \in \Delta} X_{i}$.

- $X_{i} \cap X_{j}=\varnothing \quad$ para todo $i \neq j$.

Por una cuestión de completitud, presentaremos el Axioma de Elección. La literatura sobre el mismo es prácticamente inabarcable y sus consecuencias sobre la teoría de conjuntos sigue dando discusión entre los matemáticos. Existen múltiples formas de enunciarlo, nosotros presentaremos una particular (pueden encontrarse definiciones y resultados equivalentes en (Ivorra, 2018b, 2018a; Rubin y Rubin, 1963).

Definición 2.8 (AE). $\forall X \exists F\left(F: X \rightarrow \bigcup_{V \in X} V \wedge \forall U \in X(U \neq \varnothing \rightarrow F(U) \in U)\right)$.

La anterior definición puede expresarse de la siguiente manera: sea $X$ una familia arbitraria de conjuntos no vacíos de cualquier cardinalidad, esto es, cada elemento de $X$ es a su vez un conjunto y no hay restricción sobre la cantidad de elementos de ninguno de los conjuntos. Entonces, para esa familia genérica de conjuntos $X$, existe una función de elección $F$ con dominio $X$, tal que para cada conjunto $V$ perteneciente a esta familia, la función de elección elige un elemento de este conjunto. Este axioma es crucial para la demostración de muchos resultados importantes, como la existencia de bases en espacios vectoriales, la existencia de clausura algebraica, el teorema de Tychonoff sobre la compacidad de un producto de espacios topológicos, el teorema de Hann-Banach sobre extensión de funcionales lineales, etc. Su 'virtud' es poder dar cuenta de conjuntos que no pueden ser mostrados o construidos explícitamente debido a limitaciones del lenguaje formal de la teoría de conjuntos ZF. Existen otras teorías de conjuntos (Nuevos Fundamentos de Quine) donde AE no es válido (ver (Ivorra, 2018b)).

2.3. El Hotel de Hilbert. El Hotel de Hilbert es una abstracción matemática que pone de manifiesto algunas curiosidades que tiene el concepto de infinito. Se cuenta que David Hilbert, padre del formalismo matemático, utilizaba la idea de un hotel infinito para conversar sobre temas de infinitud con alumnos y colegas. Existen muchas variantes de 
problemas relacionadas con el hotel, pero nosotros contaremos las más clásicas. Uno de los objetivos de este artículo es proponer soluciones alternativas para uno de sus casos.

Se cuenta que dos socios (uno podría ser Hilbert) tenían como objetivo crear el hotel más grande del mundo y se debatían cuántas habitaciones debía tener el mismo. Primero pensaron que 10.000 habitaciones sería un número más que pertinente, pero pronto se dieron cuenta de que tan rápido como la población mundial superara un cierto número de habitantes, su hotel correría riesgo de quedar pequeño. Querían garantizarse que ningún otro hotel pudiese tener más habitaciones que el suyo. Pronto la solución apareció en sus cabezas: el número de habitaciones no podía ser finito, es decir, el hotel debía contar con infinitas habitaciones.

Observación: En todo nuestro trabajo estamos tomando la propiedad de ser infinito como equivalente a la de no ser finito. Esto es, decimos que un conjunto es infinito si y sólo si no es equipotente con ninguna sección de los Naturales $\{1,2,3,4, \ldots, n\}$ (sin importar qué número natural $n$ se tome). No nos detendremos aquí, pero existen muchas definiciones de infinito y no siempre son equivalentes (para adentrarse en el tema puede verse (Ortiz, 1994)).

Pronto el hotel se hizo muy famoso y turistas de todas las regiones del planeta colmaban sus salas. A pesar de la infinitud del conjunto de habitaciones, el hotel tenía la siguiente regla: la administración del hotel podía disponer cambiar de habitación a cualquiera de sus huéspedes. Un día pasó algo inesperado; llegó un nuevo turista para hospedarse en el Hotel de Hilbert y el recepcionista tuvo que informarle que todas las habitaciones estaban ocupadas. Antes de que el hombre tuviese tiempo de decepcionarse, Hilbert se comunicó simultáneamente con todas las habitaciones pidiendo que cada uno de los turistas pase a la pieza siguiente, es decir, quien se encontrara en la pieza número $n$, pase a la $n+1$. De esta manera, cada uno de los turistas pasó a la pieza contigua y la pieza número 1 quedó vacía para que ingresara el nuevo viajero. Esto deja en evidencia que si tenemos un conjunto infinito y le sumamos 1, seguimos teniendo un conjunto igualmente infinito. Si lo relacionamos con las biyecciones anteriormente presentadas, diríamos que el conjunto $X=\{1,2,3,4,5,6,7, \ldots, n, n+1, \ldots$,$\} es equipotente con el$ conjunto $Y=\{2,3,4,5,6,7,8, \ldots, n+1, n+2, \ldots$,$\} . La función biyectiva que los relaciona$ es $f: X \rightarrow Y$, tal que $f(x)=x+1$.

A partir de ese momento, si llegaban $m$ nuevos viajeros al hotel, lo único que se hacía era comunicarle a cada huésped que sumara $m$ al número de su pieza, y luego pasara a esa nueva habitación. La biyección es este caso sería

$$
f(x)=x+m .
$$

Moraleja: el conjunto de los números naturales a partir del $m$ (para cualquier $m$ natural) es equipotente con el conjunto de todos los números naturales.

Pero un nuevo desafío azotó los lindes del hotel cuando por primera vez llegó un contingente infinito de turistas mientras el hotel aún estaba ocupado en su totalidad. ¡No podemos sumarle infinito a cada número de habitación! Sus habitantes pasarían sus vidas caminando los pasillos del Hotel sin llegar nunca a ningún lugar. ¿Cómo haría Hilbert y sus administradores para resolver el problema? ¡Fácil!, Hilbert ordenó a cada persona 
de su hotel que multiplique el número de su pieza por 2 y pase a ubicarse en la nueva numeración. Como la cantidad de pares es igual a la cantidad de números naturales, luego de multiplicar por 2, todos los personajes pasarían a ocupar una pieza con número par quedando todas las impares desocupadas para el ingreso del nuevo contingente. La biyección involucrada: $f: X \rightarrow Y$, tal que $f(x)=2 x$.

El último desafío hotelero consiste en albergar infinitos de estos contingentes, cada uno infinito, cuando el hotel se encuentra al ciento por ciento de ocupación. La solución estándar (El hotel infinito de Hilbert, 2021) consiste en: "El recepcionista permaneció inmutable, por lo cual tomó tranquilamente el micrófono y se comunicó solamente con las habitaciones cuyo número fuera primo o alguna potencia de estos $\left(p^{n}\right)$, les pidió que elevaran el número 2 al número de la habitación en la que se encontraban $\left(2^{p^{n}}\right)$ y se cambiaran a esa habitación. Entonces asignó a cada una de las excursiones un número primo (distinto de 2), a cada uno de los turistas de cada una de las excursiones un número par o impar $(t)$, de manera que la habitación de cada uno de los turistas, se calculaba tomando el número primo de su excursión $(p)$ y elevarlo al número que les tocó dentro de su excursión $(t)$ lo que da $p^{t} "$. Existiendo un número infinito de números primos y un número infinito de números (pares e impares), fácilmente se logró hospedar a un número infinito de infinitos huéspedes dentro de un hotel que sólo tiene un número infinito de habitaciones.

Lo anterior muestra que sumarle infinitas veces algo infinito puede no aumentar la infinitud de un conjunto. Explicitar la biyección es en este último caso un poco más complejo. Nosotros tenemos como uno de los objetivos del trabajo proponer varias soluciones alternativas a este último problema del Hotel de Hilbert.

\section{§3. Particiones de los Naturales}

Como nombramos anteriormente, toda partición de un dominio define una relación de equivalencia sobre el mismo y viceversa (Apostol, 1984; Vinogradov, 1977). Nosotros generaremos las particiones y obtendremos, por lo tanto, las relaciones de equivalencia asociadas. Para comenzar, mostraremos cómo generar una de las al menos numerables PDN. Para que una partición de los Naturales pueda ser llamada doblemente numerable, es necesario y suficiente que cada uno de los numerables subconjuntos que componen la partición del dominio (no puede ser una partición finita) sea numerable, es decir, equipotente con $\mathbb{N}$, que sean disjuntos dos a dos (su intersección sea el conjunto vacío) y que al unirlos recobremos todos los Naturales.

Consideremos los números naturales con su orden habitual comenzando por el 1, pero el razonamiento no cambia si tomamos el 0 como natural. También podríamos extender este proceso a los números enteros.

Comencemos con la serie natural:

$12234456 \quad 7891011121314151617$

$18192021222324252627282930313233 \ldots$

Con los colores simplemente explicitamos el conjunto al que haremos pertenecer cada natural, es decir, nuestra proceso comienza generando conjuntos sucesivos de longitud 
doble que la anterior comenzando por una longitud inicial 2:

$$
\begin{gathered}
C_{1}=\{1,2\}, \quad C_{2}=\{3,4,5,6\}, \quad C_{3}=\{7,8,9,10,11,12,13,14\}, \\
C_{4}=\{15,16,17,18,19,20,21,22,23,24,25,26,27,28,29,30\},
\end{gathered}
$$

etcétera. Está claro que

$$
\mathbb{N}=\bigcup_{\ell=1}^{\infty} C_{\ell}, \quad C_{\ell} \cap C_{\ell^{\prime}}=\varnothing, \quad \ell \neq \ell^{\prime} .
$$

Observación: Notemos que $\operatorname{los} C_{\ell}$ forman una partición de los Naturales, pero tal partición no es doblemente numerable. Esto se debe a que, a pesar de existen tantos $C_{\ell}$ como números naturales (y son disjuntos), los mismos no son numerables (son todos finitos).

El siguiente paso consiste en elegir el primer elemento de cada uno de los conjuntos $C_{\ell}$ para formar el conjunto $A_{1}$. Esto se puede hacer de manera algorítmica gracias a que los Naturales están bien ordenados. Tenemos entonces:

$$
A_{1}=\{1,3,7,15,31, \ldots\} .
$$

Donde los elementos de $A_{1}$ mantienen la siguiente recursión:

$$
a_{1, i+1}=2 a_{1, i}+1, \quad a_{1,1}=1 .
$$

Cada elemento de este conjunto es igual al sucesor del doble de su anterior. Además, es claro que es un conjunto infinito numerable, ya que existen infinitos $C_{\ell}$ y cada uno tiene un primer elemento.

Ahora construimos el siguiente conjunto, $A_{2}$, tomando el segundo elemento de cada $C_{\ell}$. Esto es equivalente a sacar de los conjuntos originales el primer elemento ya utilizados en $A_{1}$ $\mathrm{y}$ volver a tomarle el primer elemento. Esto es algo que puede realizarse sin inconveniente de forma recursiva.

$$
A_{2}=\{2,4,8,16,32, \ldots\} .
$$

Donde sus elementos mantienen la siguiente relación:

$$
a_{2, i+1}=2 a_{2, i}, \quad a_{2,1}=2 .
$$

Obtenemos un nuevo conjunto numerable donde cada elemento es el doble de su anterior.

Al formar el tercer conjunto, debemos tomar el tercer elemento de cada uno de los $C_{\ell}$. Observamos directamente que $C_{1}$ no tiene tercer elemento, con lo cual comenzamos por el conjunto siguiente, $C_{2}$, que es el primer conjunto que tiene tercer elemento.

$$
\begin{gathered}
A_{3}=\{5,9,17,33, \ldots\} \\
a_{3, i+1}=2 a_{3, i}-1, \quad a_{3,1}=5 .
\end{gathered}
$$

En este caso, cada elemento se genera a partir del anterior duplicándolo y restando 1.

Siguiendo de esta manera:

$$
\begin{gathered}
A_{4}=\{6,10,18,34, \ldots\} \\
a_{4, i+1}=2 a_{4, i}-2, \quad a_{4,1}=6 .
\end{gathered}
$$


Para el caso siguiente, hay que notar que los dos primeros $C_{\ell}$ no cuentan con quinto elemento por lo que el primer elemento de $A_{5}$ pertenecerá a $C_{3}$, el primero de estos conjuntos que tiene un quinto elemento, el 11.

$$
\begin{gathered}
A_{5}=\{11,19,35,67, \ldots\} \\
a_{5, i+1}=2 a_{5, i}-3, \quad a_{5,1}=11 .
\end{gathered}
$$

Queda claro de qué forma continuar nuestra construcción y, además, que la misma se puede hacer tomando como base cualquier número mayor que 1, en vez de base 2 como hemos elegido para mostrar el proceso de construcción. Más adelante, nos centraremos en analizar la cantidad de formas diferentes de realizar esta tarea. Por construcción, se cumple que:

$$
\mathbb{N}=\bigcup_{n=1}^{\infty} A_{n}, \quad A_{n} \cap A_{n^{\prime}}=\varnothing, \quad n \neq n^{\prime} \quad \text { y } \quad \forall n\left|A_{n}\right|=|\mathbb{N}|=\aleph_{0}
$$

Es fácil probar que cada número natural pertenece sólo a uno de los $A_{n}$. Esto se debe a que cada natural pertenece a un único $C_{\ell}$ (son disjuntos) y que dentro de ese conjunto puede ocupar una sola ubicación, la $n$-ésima. Cuando mostremos el caso general, se verá que debido a cómo se calcula el $n$ asociado con cada número natural, tener el mismo $n$ genera una relación que es reflexiva, transitiva y simétrica, es decir, genera una relación de equivalencia.

Por lo tanto, tenemos a los números naturales separados en una cantidad numerable de conjuntos disjuntos cada uno equipotente con $\mathbb{N}$. Esta partición de los naturales es un ejemplo de lo que llamamos partición doblemente numerable (PDN) y tendrá su correspondiente relación de equivalencia asociada.

Volvamos al Hotel de Hilbert. Si llegaran simultáneamente numerables contingentes cada uno numerable (como en el último caso brindado en la sección 2), tenemos de qué manera asignar una pieza a cada persona. Esta manera, no sólo es una alternativa diferente a la solución clásica mostrada, sino que genera infinitas alternativas equivalentes más. Indexamos con $n$ al contingente en cuestión, asignémosle la clase $A_{n}$ junto con un índice $i$ que denote su posición dentro del $A_{n}$. Como existirá un único natural asociado a cada par $(n, i)$, cada individuo contará con su propia pieza. Cada persona pertenece a un único contingente, lo que determina unívocamente el $n$, y tiene un orden dado $(i)$ dentro del mismo, que también es único. Por lo tanto, cada persona tiene un único par $(n, i)$. Si el hotel ya estuviese ocupado en su totalidad cuando llegan los infinitos contingentes de turistas, entonces dejamos libre todas las piezas impares (con el ardid clásico del problema) y realizamos la partición mostrada sobre los impares.

Antes de pasar a explicitar el formalismo recursivo que fundamenta esta partición, ya podemos notar que nos hemos creado un ejemplo particular de numerables conjuntos disjuntos de a pares, cada uno numerable, cuya unión es numerable (ya que su unión es el conjunto $\mathbb{N}$ ). Tenemos a mano un ejemplo concreto para mostrar cuando estemos comenzando a explicar temas sobre cardinalidad transfinita. Con el ejemplo clásico del hotel de Hilbert, quedaba a la vista que la unión de dos conjuntos numerables era otro conjunto de la misma cardinalidad, pero ahora podemos mostrar un ejemplo que se compromete 
con una cantidad numerables de los mismos. Por supuesto, que esto es sólo un ejemplo en particular, pero explicita una propiedad interesante de los conjuntos numerables.

\section{§4. Formalización del problema}

Vamos a mostrar la función recursiva que asigna de forma biunívoca un $n$ y un $i$ a cada número natural $x$. El $n$ determinará a qué clase $\left(A_{n}\right)$ pertenece y el $i$ designará su posición dentro de la misma. Esto es, dado $x \in \mathbb{N}$, asignaremos una única clase $A_{n}$ y dentro de esta clase numerable, la posición $i$-ésima. Esta será una forma posibles, entre muchas, de asignar biyectivamente un par $(n, i)$ a cada $x$ natural, lo que muestra la relación que guarda el problema de generar particiones doblemente numerables (y unir numerables conjuntos disjuntos) con el de biyectar $\mathbb{N}$ con $\mathbb{N} \times \mathbb{N}$.

Todo número natural $x$ mayor que 2 se puede acotar de la siguiente manera (el caso $x=1$ y $x=2$ no generan inconveniente, ya que sabemos cómo ubicarlos):

$$
\sum_{j=1}^{m} 2^{j}<x \leq \sum_{j=1}^{m+1} 2^{j}
$$

La condición anterior puede expresarse equivalentemente como

$$
2^{m+1}-2<x \leq 2^{m+2}-2 .
$$

Utilizamos más la primera forma al hacer los cálculos siguientes porque evidencia mejor el razonamiento secuencial y constructivo que utilizamos.

Según la ecuación (4.1), cada natural define de manera unívoca un $m$. Puede verse que $\ell=m+1$, donde $\ell$ es el subíndice de los conjuntos $C_{\ell}$ del principio. Definimos el número $n$ de la clase $A_{n}$ asociada con $x$ de la siguiente manera:

$$
n=x-\sum_{j=1}^{m} 2^{j}=x-\left(2^{m+1}-2\right)=x-2^{m+1}+2,
$$

donde $m$ está determinado por la ecuación (4.1).

Veamos un ejemplo: si $x=17$, entonces

$$
2+4+8<17 \leq 2+4+8+16 .
$$

Usando (4.1) tenemos que $m=3 \mathrm{y} \ell=4$. Con (4.2) calculamos que $n=17-2^{4}+2=3$. Por lo tanto, $17 \in A_{3}$, resultado que concuerda con nuestra definición de $A_{3}$. Falta ahora que determinemos qué ubicación ocupa dentro de esta clase. La ubicación $i$-ésima dentro de $A_{n}$ viene dada por:

$$
i=\log _{2}\left(\frac{x-n+2}{a_{n, 1}-n+2}\right)+1 .
$$

Donde $a_{n, 1}$ es el primer elemento del conjunto $A_{n}$ y viene dado por:

$$
a_{n, 1}=n+\sum_{j=1}^{k} 2^{j}=2^{k+1}+n-2 .
$$


Donde $k$ debe satisfacer:

$$
2^{k}<n \leq 2^{k+1} .
$$

El número $k$ puede ser nulo o negativo $(-1)$. Si $n=1$, entonces $k=-1$ :

$$
k \in\{-1,0,1,2,3,4,5, \ldots\} .
$$

Esto no genera problema en la ecuación (4.4) (al calcular el primer elemento de ese conjunto) si tomamos la convención de que el resultado de la sumatoria es nulo cada vez que el índice superior es menor que el inferior. Veamos ahora el funcionamiento completo de este procedimiento algorítmico. Supongamos tener $x=131$ y veamos qué ubicación le corresponde dentro de su clase $A_{n}$ correspondiente. Primero debemos obtener el $n$ con las ecuaciones (4.1) y (4.2):

$$
2+4+8+16+32+64<131 \leq 2+4+8+16+32+64+128
$$

entonces

$$
m=6 \quad \text { y } \quad n=131-126=5 .
$$

Es decir, $131 \in A_{5}$. Usando las ecuaciones (4.3), (4.4) y (4.5) calculamos el $i$ asociado. Por (4.5) tenemos:

$$
2^{2}<5 \leq 2^{3} .
$$

Concluyendo que $k=2$. Usando este $k$ en (4.4):

$$
a_{5,1}=5+\sum_{j=1}^{2} 2^{j}=11 .
$$

Con lo que obtenemos el primer elemento de esta clase de equivalencia. Finalmente, por (4) llegamos a:

$$
i=\log _{2}\left(\frac{131-5+2}{11-5+2}\right)+1=5
$$

Por lo tanto, 131 es el quinto elemento de $A_{5}$. En relación al hotel, nuestro resultado dice que al quinto turista del contingente número 5 debemos asignarle la pieza número 131. Aunque de esta manera lo que hicimos fue asignar una habitación a un turista, podemos hacer el procedimiento inverso como mostraremos a continuación. Si quisiésemos, por ejemplo, ver qué pieza asignar al sexto turista del contingente 3, lo que tenemos que hacer es ver qué numero natural ocupa la posición 6 en $A_{3}$. Debemos calcular el primer elemento de esta clase y luego recursivamente los demás elementos a través de la recursión que caracteriza a $A_{3}$, en este caso, al doble del elemento anterior tomarle sucesor. La relación general de recursión que se encuentra detrás de todos los $A_{n}$ es la siguiente:

$$
a_{n, i+1}=2^{i} a_{n, 1}-(n-2)\left(2^{i}-1\right) .
$$

Donde tanto $n$ como $i$ se toman a partir de 1 y cada $a_{n, 1}$ se calcula según (4.4). Esta función es la inversa de la que a cada $x$ le asigna un par $(n, i)$. Veamos que esto se condice con los primeros $A_{n}$ que mostramos al comienzo. Si $n=1$, entonces

$$
a_{1, i+1}=2^{i} a_{1,1}+\left(2^{i}-1\right) .
$$


Que genera la sucesión $1,3,7,15, \ldots$ a partir de su primer elemento, $a_{1,1}=1$. Si $n=2$,

$$
a_{2, i+1}=2^{i} a_{2,1} \text {. }
$$

Para $n=3$,

$$
a_{3, i+1}=2^{i} a_{3,1}-\left(2^{i}-1\right) .
$$

Y de esta manera puede corroborarse para el resto de los números.

Volviendo al hotel, estábamos interesados en ver qué habitación darle al sexto integrante del contingente 3. Calculemos primero $a_{3,1}$. Según la ecuación (4.5),

$$
2^{1}<3 \leq 2^{2}
$$

implica que $k=1$. Entonces, por (5):

$$
a_{3,1}=3+\sum_{j=1}^{1} 2^{j}=5 .
$$

Y finalmente obtenemos (por (4.6)):

$$
a_{3,6}=2^{5} \cdot 5-\left(2^{5}-1\right)=129 .
$$

Por lo tanto, le otorgaremos la habitación 129 al viajero en cuestión. Llegado este punto, queda a la vista que el razonamiento que hemos hecho puede realizarse usando otro número como base, es decir, podríamos realizar un procedimiento análogo tomando desde el principio los $C_{l}$ con longitudes que sean potencias de 3 (no deben ser necesariamente potencias). Cualquier número natural mayor que 1 cumple el objetivo. También puede probarse que podríamos usar como base números racionales, como por ejemplo el $\frac{3}{2}$. Estas alternativas las analizaremos más adelante. Aunque estamos relacionando las PDN con el Hotel de Hilbert, tenemos también como objetivo generar tantos ejemplos como podamos (de forma recursiva) de colecciones numerables de conjuntos numerables y disjuntos cuya unión sea numerable. Los $A_{n}$ cumplen este objetivo para cualquier base que sea mayor que 1 (no decimos que esto no pueda realizarse con números menores, sólo que nuestro procedimiento está pensado par bases mayores). Algo esencial para nuestro procedimiento es que la suma de las longitudes de los conjuntos $C_{\ell}$ no esté acotada. Por lo tanto, tenemos al menos numerables ejemplos, que podemos generar de forma efectiva, que muestran esta propiedad de los conjuntos numerables con respecto a la cardinalidad de su unión. La relación de equivalencia que está detrás de todas estas particiones, al margen de la base que la genere, es la siguiente: dos naturales $x$, y están relacionados si y sólo si pertenecen al mismo $A_{n}$, es decir, si tienen asociado el mismo $n$ en (4.2). Tener asignado el mismo $n$ se prueba que es una relación reflexiva, simétrica y transitiva.

Mostraremos ahora las ecuaciones que caracterizan a la partición asociada a base igual a 3 para explicitar otro ejemplo de esta forma de razonar.

Las ecuaciones que determinan el número natural asociado con $n$ e $i$ dados son:

$$
\begin{gathered}
a_{n, i+1}=3^{i} a_{n, 1}-\frac{1}{2}(2 n-3)\left(3^{i}-1\right), \\
a_{n, 1}=n+\sum_{j=1}^{k} 3^{j}, \quad 3^{k}<n \leq 3^{k+1} .
\end{gathered}
$$


Estas ecuaciones generan la partición siguiente:

$$
\begin{gathered}
A_{1}=\{1,4,13,40, \ldots\} \\
A_{2}=\{2,5,14,41, \ldots\} \\
A_{3}=\{3,6,15,42, \ldots\} \\
A_{4}=\{7,16,43,124, \ldots\} \\
A_{5}=\{8,17,44,125, \ldots\}
\end{gathered}
$$

Para una base $b \in \mathbb{N} \backslash\{1\}$ las ecuaciones son:

$$
\begin{aligned}
a_{n, i+1} & =b^{i} a_{n, 1}-((b-1) n-b)\left(\frac{b^{i}-1}{b-1}\right), \\
a_{n, 1} & =n+\sum_{j=1}^{k} b^{j}, \quad b^{k}<n \leq b^{k+1} .
\end{aligned}
$$

En la siguiente página puede descargarse el programa que realiza la partición correspondiente en base 2. Dado un $x$ natural, asigna los correspondientes $n$ e $i$, además de mostrar algunos elementos del $A_{n}$ en cuestión, y si se ingresa el par $(n, i)$, devuelve el natural que corresponde según nuestras ecuaciones: https://drive.google.com/file/d/OB-rDGOh8gCl2OE1RdE5BVGhac1E/ view

\section{§5. Cardinalidad de las PDN}

Como hemos probado en la sección anterior, tenemos al menos una forma de realizar estas particiones por cada número natural mayor o igual que 2, es decir, existen al menos numerables particiones de los números naturales en numerables clases, cada una numerable, disjuntas de a pares. Además, en los naturales estas particiones se pueden generar de forma algorítmica. Veamos ahora que, en realidad, existe una de estas particiones por cada número real mayor que 1. Puede apreciarse en las construcciones mostradas que no hemos utilizado propiedades de la base que sean propiamente exclusivas de los números naturales. La única propiedad indispensable es que los naturales queden incluidos en la unión de todos $\operatorname{los} C_{\ell}$, que estos conjuntos sean disjuntos, y que en la sucesión de las longitudes $C_{\ell}$ sea estrictamente creciente (aunque si en vez de estrictamente creciente pidiéramos que fuera no acotada, podríamos aplicar el método con pequeñas modificaciones). Para explicitar un poco esto, realizaremos la siguiente construcción.

Si mostramos que existe una PDN por cada número real mayor que 2, entonces habrá al menos tantas PDN como número reales, ya que el conjunto $[2,+\infty)$ es equipotente con $\mathbb{R}$. Sin embargo, nuestro procedimiento efectivo general para generar PDN puede aplicarse para cualquier número real mayor estricto que 1. Por esto mismo, el ejemplo que presentaremos al terminar la siguiente construcción toma como base $\sqrt{2}$. Tomar una base mayor o igual que 2 simplemente sirve para visualizar mejor el proceso, ya que las potencias de 2 crecen de una forma rápida y esto produce que entre una potencia y la siguiente siempre exista al menos un natural facilitando el procedimiento de cálculo. 
Sea $r \in \mathbb{R}$, tal que $2 \leq r$. Definimos los $C_{\ell}$ de la siguiente forma:

$$
C_{\ell}=\left[r^{\ell-1}, r^{\ell}\right), \quad 1 \leq \ell .
$$

Entonces,

$$
\mathbb{N} \subset \bigcup_{\ell=1}^{\infty} C_{\ell}, \quad C_{\ell} \cap C_{\ell^{\prime}}=\varnothing, \quad \ell \neq \ell^{\prime} .
$$

Los $A_{n}$ los definimos como

$$
A_{n}=\left\{x \in \mathbb{N}:\left[x-r^{\ell-1}\right]+1=n, x \in C_{\ell}, 1 \leq \ell\right\} .
$$

Donde [.] denota la función parte entera. Todos los $A_{n}$ a partir de un momento (a partir de un $\ell_{0}$ ) tienen exactamente un elemento de cada $C_{\ell}$, lo que significa que cada uno de estos conjuntos es numerable. También es fácil ver que son disjuntos de a pares. Para mostrar que cada real mayor o igual que 2 genera una partición diferente, hay que ver que dados dos reales cualesquiera, $r$ y $r^{\prime}$, sus potencias difieren en al menos una unidad a partir de un momento. Si esto pasa, a partir de ese momento, los $C_{\ell}$ se distinguirán de los $C_{\ell^{\prime}} \mathrm{y}$ esa diferencia repercutirá (por definición) en los $A_{n}$ (a partir de un momento). En otras palabras, aunque dos números reales $r$ y $r^{\prime}$ sean muy cercanos, existirá una potencia natural $n_{0}$ a partir de la cual la diferencia entre $r^{n_{0}}$ y $\left(r^{\prime}\right)^{n_{0}}$ será mayor que 1. Y esto garantizará que exista un natural entre ambos.

También podríamos preguntarnos, ¿todo número natural pertenece a algún $A_{n}$ ?

Veamos esto detenidamente. Como $\mathbb{N} \subset \cup_{\ell=1}^{\infty} C_{\ell}$ y los $C_{\ell}$ son disjuntos, dado un número natural $n_{1}$, existe un $\ell_{1}$, tal que $n_{1} \in C_{\ell_{1}}=\left[r^{\ell_{1}-1}, r^{\ell_{1}}\right)$. Formemos ahora el siguiente número natural $n$,

$$
n=\left[n_{1}-r^{\ell_{1}-1}\right]+1 .
$$

Mirando la ecuación 5.1, vemos que los números $\ell_{1}, n$ conducen directamente a que $n_{1} \in A_{n}$. Con lo que queda probado de qué manera calcular el único $A_{n}$ al cual pertenece cualquier natural.

Mostraremos, como bien habíamos ya anticipado, un ejemplo de aplicación con una base mayor que 1 , pero menor que 2 . Tomemos $r=\sqrt{2}$ y veamos qué partición de los naturales nos genera esta base.

Primero generamos los $C_{\ell}$.

$$
\begin{array}{ll}
C_{1}=\left[\sqrt{2}^{0}, \sqrt{2}^{1}\right)=[1, \sqrt{2}), & C_{2}=\left[\sqrt{2}^{1}, \sqrt{2}^{2}\right)=[\sqrt{2}, 2), \\
C_{3}=\left[\sqrt{2}^{2}, 2 \sqrt{2}\right)=[2,2 \sqrt{2}), & C_{4}=\left[2 \sqrt{2}, \sqrt{2}^{4}\right)=[2 \sqrt{2}, 4), \\
C_{5}=\left[\sqrt{2}^{4}, 4 \sqrt{2}\right)=[4,4 \sqrt{2}), & C_{6}=\left[4 \sqrt{2}, \sqrt{2}^{6}\right)=[4 \sqrt{2}, 8), \ldots
\end{array}
$$

Observación: El inconveniente que trae una base mayor que 1, pero menor que dos, es que podemos tener algunos $C_{\ell}, C_{2}$ en este caso, que no tienen ningún natural. Son conjuntos superfluos para el método.

Por lo tanto,

$$
A_{1}=\left\{x \in \mathbb{N}:\left[x-\sqrt{2}^{\ell-1}\right]+1=1, x \in C_{\ell}, 1 \leq \ell\right\} .
$$


Para cada valor de $l$ natural, obtenemos como máximo un elemento para $A_{1}$. Puede pasar que para algún $l$ no haya ningún número natural que pertenezca a $A_{1}$ como bien observamos anteriormente. En este caso, $C_{2}$ no tiene ningún natural, por lo tanto no aportará en la construcción de ninguno de los $A_{n}$. Con lo cual nos queda

$$
\begin{gathered}
A_{1}=\{1,2,3,4,6,8,12,16,23, \ldots\}, \\
A_{2}=\left\{x \in \mathbb{N}:\left[x-\sqrt{2}^{\ell-1}\right]+1=2, x \in C_{\ell}, 1 \leq \ell\right\}=\{5,7,9,13,17,24, \ldots\}, \\
A_{3}=\left\{x \in \mathbb{N}:\left[x-\sqrt{2}^{\ell-1}\right]+1=3, x \in C_{\ell}, 1 \leq \ell\right\}=\{10,14,18,25, \ldots\} .
\end{gathered}
$$

Y de esta manera se puede continuar para todo número natural.

En los modelos estándar de los números reales, siempre tenemos asegurado que dos números reales distintos tengan potencias diferentes a partir de un momento, y que esta diferencia sea mayor que 1 garantizándose la buena definición de nuestra partición. Esto es una consecuencia del binomio de Newton y de la Arquimeneidad de los reales (estándar) (Fava y Zó, 2013; Stein y Shakarchi, 2007).

Si tenemos dos números reales $r, r^{\prime}$ tan cercanos como se quiera. Entonces,

$$
r^{\prime}=r+\epsilon, \quad \epsilon>0 .
$$

Y por el binomio de Newton, tenemos entonces:

$$
\begin{aligned}
\left(r^{\prime}\right)^{m}=(r+\epsilon)^{m} & =\sum_{k=0}^{m} \frac{m !}{k !(m-k) !} r^{m-k} \epsilon^{k} \\
& =r^{m}+\frac{m !}{(m-1) !} r^{m-1} \epsilon+\sum_{k=2}^{m} \frac{m !}{k !(m-k) !} r^{m-k} \epsilon^{k} .
\end{aligned}
$$

Para cualquier $\epsilon$ fijo dado (y $r$ mayores que uno), el término $\frac{m !}{(m-1) !} r^{m-1} \epsilon$ puede hacerse, a partir de un cierto $m_{0}$, tan grande como se desee. Esto se debe a la propiedad de Arquimediana nombrada. Por lo tanto, a partir de un momento este término supera la unidad y se obtiene lo deseado, ya que el resto de los términos son todos positivos.

Observación: Dentro de los modelos no estándar de los reales, los elementos pertenecientes al halo de cada real podrían no cumplir con lo anterior (ver (Cobillón, 2015)).

Por lo tanto, este método permite ver que la cardinalidad de las PDN es mayor que la de los naturales. También podemos ver que no puede superar el cardinal de los reales, ya que cada una de estas particiones pertenece a $\mathcal{P}_{\text {num }}\left(\mathcal{P}_{\text {num }}(\mathbb{N})\right)$, donde

$$
\mathcal{P}_{\text {num }}(\mathbb{N})=\{A \subset \mathbb{N}:|A|=|\mathbb{N}|\} .
$$

Se puede probar (usando AE) que las partes numerables de los naturales tienen la potencia del continuo. Por otro lado, las partes numerables de los reales también tienen la misma potencia. Por lo tanto, la cantidad de PDN no puede ser mayor que la cantidad de reales. Hemos probado que estas particiones de los naturales tienen la potencia de los números reales.

Antes de terminar la sección, podría ser de interés presentar una prueba, hasta donde nosotros sabemos, original de uno de los resultados que acabamos de nombrar: Que 
las partes numerables de los naturales tiene la misma potencia que las partes de los naturales. Presentamos la prueba como apoyo a nuestros razonamientos. La realizaremos para un caso general que incluye el caso de los números naturales.

Observación: El resultado obtenido brinda una forma alternativa de caracterizar a los conjuntos infinitos, que se prueba equivalente a las demás definiciones si se utiliza AE.

Definimos

$$
\mathcal{P}_{\text {max }}(A)=\{D \subset A:|D|=|A|\} .
$$

Teorema 5.1. Sean $A, B, C$ conjuntos, tales que $A=B \cup C, B \cap C=\varnothing y|A|=|B|=|C|$. Bajo estas condiciones, $\left|\mathcal{P}_{\text {max }}(A)\right|=|\mathcal{P}(A)|$.

Está claro que esto se cumple en el caso particular de los naturales, ya que la partición en pares e impares sirve para cumplir las condiciones del teorema y, por otro lado, sabemos que las partes numerables de los naturales son equipotentes con las partes de los naturales (ambos conjuntos tienen la cardinalidad de los reales).

Demostración. Comencemos formando el siguiente conjunto:

$$
\{F \cup C: F \subset B\} \text {. }
$$

Claramente pasa que

$$
\{F \cup C: F \subset B\} \subset \mathcal{P}_{\max }(A),
$$

ya que la cardinalidad de $F \cup C$ es igual a la cardinalidad de $C$.

Observación: Esto es debido a que $C$ tiene la cardinalidad del conjunto $A$ (que es la máxima posible para el caso). El conjunto $C$ es unido a cada $F$ de forma intensional para que cada elemento de $\{F \cup C: F \subset B\}$ tenga la cardinalidad máxima (y de esta manera pertenezca también a $\mathcal{P}_{\max }(A)$ ). Por otro lado, este conjunto tendrá tantos elementos como conjuntos posibles haya en $B$, uno por cada $F$. Para garantizar lo anterior, necesitamos una de las consecuencia del teorema (CSB) (2.5), más precisamente una versión más débil de (CSB) (debida a Dedekind) que dice que si $A$ es equipotente con un subconjunto de $C^{\prime}$ y $C^{\prime} \subset A$, entonces $C^{\prime}$ es equipotente con $A$.

Por lo tanto, si se da la inclusión anterior, tenemos que

$$
|\{F \cup C: F \subset B\}| \leq\left|\mathcal{P}_{\max }(A)\right| .
$$

Por otro lado, por construcción, este conjuntos tiene exactamente un elemento por cada subconjunto de $B$, con lo cual tenemos que

$$
|\mathcal{P}(B)|=|\{F \cup C: F \subset B\}| \leq\left|\mathcal{P}_{\max }(A)\right| .
$$

Y como $A$ y $B$ son equipotentes, entonces

$$
|\mathcal{P}(A)|=|\mathcal{P}(B)|=|\{F \cup C: F \subset B\}| \leq\left|\mathcal{P}_{\max }(A)\right| .
$$

De la cadena anterior puede concluirse que

$$
|\mathcal{P}(A)| \leq\left|\mathcal{P}_{\max }(A)\right| .
$$


Pero por definición de $\mathcal{P}_{\max }$ se tiene que

$$
\left|\mathcal{P}_{\text {max }}(A)\right| \leq|\mathcal{P}(A)| .
$$

Utilizando la antisimetría de la relación tener cardinal menor o igual que, justificada por el Teorema 2.5 (CSB), y juntando las últimas dos desigualdades, obtenemos

$$
\left|\mathcal{P}_{\text {max }}(A)\right|=|\mathcal{P}(A)|
$$

como queríamos ver.

Para el caso particular de los números naturales, nuestro resultado se expresa como

$$
\left|\mathcal{P}_{\text {num }}(\mathbb{N})\right|=|\mathcal{P}(\mathbb{N})|=2^{\aleph_{0}} .
$$

Siempre que un conjunto dado pueda disjuntarse en (al menos) dos conjuntos equipotentes, entonces los conjuntos de máxima cardinalidad son aquellos que aportan a la cardinalidad del conjunto de sus partes. Lo que caracterizaría desde este punto de vista a los conjuntos infinitos sería la siguiente propiedad:

$$
\left|\mathcal{P}_{\max }(A)\right|>|A| \text {. }
$$

Esto último significa que la cardinalidad de las partes máximas pasan de tener una cardinalidad igual a 1 (para todos los conjuntos finitos) a tener una cardinalidad $2^{\aleph_{0}}$ (para los conjuntos no finitos) sin escala intermedia. Esta propiedad característica de los conjuntos infinitos se prueba equivalente a las demás definiciones de infinitos (usando AE). Este resultado es de importancia para nosotros porque se basa en la propiedad fundamental de poder generar una partición dada. Además, si quisiéramos aplicar el mismo procedimiento utilizado con los naturales para el caso de un conjunto numerable general, el resultado obtenido sobre la cardinalidad de sus partes máximas puede ser de importancia a la hora de probar cuántas particiones diferentes podemos generar. En contraposición con la propiedad mostrada sobre las partes numerables de los Naturales, podemos nombrar que si en lugar de quedarnos con los subconjuntos infinitos (numerables) de $\mathbb{N}$, tomáramos sólo los finitos, entonces la cardinalidad de sus partes finitas, es decir, $\mathcal{P}_{\text {fin }}(\mathbb{N})$ sería la misma que la de los números naturales. En otras palabras, existen tantos subconjuntos finitos de naturales como naturales mismos, pero existen más subconjuntos infinitos de $\mathbb{N}$ que números naturales. En ecuaciones,

$$
\left|\mathcal{P}_{\text {num }}(\mathbb{N})\right|=|\mathcal{P}(\mathbb{N})|, \quad\left|\mathcal{P}_{\text {fin }}(\mathbb{N})\right|=|\mathbb{N}| .
$$

Vamos a dejar para un próximo trabajo la cuestión de bajo qué condiciones pueden darse pruebas de equivalencia entre la siguiente definición de infinito, motivada por el Teorema 5.1, y las definiciones más utilizadas en la literatura.

Definición 5.2 (Versión 1). Un conjunto $X$ es infinito si y sólo si $\left|\mathcal{P}_{\max }(X)\right|>|X|$.

Definición 5.3 (Versión 2). Un conjunto $X$ es infinito si y sólo si $\left|\mathcal{P}_{\max }(X)\right|>1$.

\section{§6. Una aplicación interesante}

Cuando usamos la ecuación (5.1) para el caso de particiones generadas por reales, nos importó que todos los naturales quedaran distribuidos en las diferentes $A_{n}$, por eso 
usábamos la parte entera en la definición. Vamos a variar ahora ligeramente la definición de los $A_{n}$ para incorporar el caso real. Dado un $r$ fijo mayor que uno, definimos los conjuntos $C_{\ell}$ como antes:

$$
C_{\ell}=\left[r^{\ell-1}, r^{\ell}\right), \quad \ell \in \mathbb{N} .
$$

Entonces,

$$
\mathbb{R}_{\geq 1} \subset \bigcup_{l=1}^{\infty} C_{\ell}, \quad C_{l} \cap C_{\ell^{\prime}}=\varnothing, \quad \ell \neq \ell^{\prime} .
$$

Para cada $d \in \mathbb{R}_{\geq 0}$, definimos $A_{d}$ como

$$
A_{d}=\left\{x \in \mathbb{R}_{\geq 1}: x-r^{\ell_{0}-1}=d \mathrm{y} x \in C_{\ell_{0}}\right\} .
$$

Por cada $C_{\ell}$ puede existir a lo sumo un $x$ cuya distancia al primer elemento del $C_{\ell}$ al que pertenece $\left(x-r^{\ell-1}\right)$ sea exactamente $d$. A partir de un cierto momento, todos los $C_{\ell}$ tienen un elemento que cumple esa condición, por lo tanto cada $A_{d}$ es numerable. También puede probarse que ningún $x$ puede pertenecer a más de un $A_{d}$ (si $r$ se encuentra fijo) y que todo real mayor que 1 pertenece a alguno de $\operatorname{los} A_{d}$.

Si un $x$ perteneciera tanto a $A_{d}$ como a $A_{d^{\prime}}$ (con $d$ y $d^{\prime}$ distintos), entonces estaría a distancia $d$ del primer elemento de algún $C_{\ell}$, al cual pertenece, y también a distancia $d^{\prime}$ del primer elemento de un $C_{\ell^{\prime}}$, al cual también pertenece. Pero como los $C_{\ell}$ son disjuntos, este no puede ser el caso. Por otro lado, como todo real mayor que 1 pertenece a un único $C_{\ell}$, la distancia al primer elemento de ese conjunto determina de forma unívoca el $A_{d}$ al que pertenece.

Los $C_{\ell}$ forman una partición de los $\mathbb{R}_{\geq 1}$ en numerables conjuntos donde cada uno es equipotente con los reales. Los $A_{d}$ forman una partición del mismo conjunto, pero en un continuo de conjuntos, identificados por $d$, donde cada uno es numerable. Las particiones del estilo $C_{\ell}$ sirven de ejemplos para la proposición que dice que la unión numerable de conjuntos con potencia del continuo da como resultado otro conjunto cuya cardinalidad es el continuo. Las particiones del estilo $A_{d}$ pueden servir de ejemplos de que la unión continua de conjuntos numerables tienen la cardinalidad del continuo. Que la cardinalidad de las partes numerables de los reales sea igual a la cardinalidad de los reales está en total acuerdo con lo que mostramos con este ejemplo.

\section{§7. Comentarios finales}

Las particiones doblemente numerables de los números naturales se presentan como un método algorítmico efectivo a la hora de generar múltiples ejemplos de conjuntos numerables y disjuntos cuya unión es numerable. Se probó que existen tantas particiones de estas como números reales y se explicitó un método efectivo para construir una PDN por cada natural mayor que 1 . Hemos presentado un ejemplo de programa que realiza esta tarea en el caso particular de base igual a 2. Su utilidad a la hora de resolver problemas de cardinalidad ha quedado a la vista con algunos ejemplos y se mostró eficiente para resolver variantes potenciadas del Hotel de Hilbert . Queda pendiente para futuras investigaciones el rol que estas particiones puedan jugar definiendo posibles modelos para los números reales 
como así también la relación directa que guardan con los números reales (no estándar) ilimitados.

Agradecimientos. Estamos muy agradecidos con Rafael Grimson por sus sugerencias y recomendaciones cuando la idea se estaba gestando. También queremos agradecerle al revisor anónimo, su experticia ayudó a dejar más claro y completo el presente artículo.

\section{Bibliografía}

Apostol, T. M. (1984). Introducción a la teoría analítica de números. REVERTE, ISBN: 9788429150063.

Apostol, T. M. (1998). Análisis matemático. REVERTE, Segunda edición. ISBN: 8429150048.

Basa, J. (2014). Sobre el Teorema de Cantor-Bernstein y la matemática de conjuntos.

Cobillón, M. S. (2015). Análisis real no estándar. Facultad de Matematiques. Universitat de Barcelona.

El hotel de Hilbert. (2020). El hotel de Hilbert. Descargado de https : / plato - stanford.edu/entries/spacetime-supertasks

El hotel infinito de Hilbert. (2021). El hotel infinito de Hilbert. Descargado de https://es.wikipedia.org/wiki/El_hotel_infinito_de -Hilbert

Fava, N., y Zó, F. (2013). Medida e integral de lebesgue. curso de grado. fascículo 4. Facultad de Ciencias Exactas y Naturales. Universidad de Buenos.

Ivorra, C. (2018a). Teoría de conjuntos y topología. Descargado de https:// www. uv.es/ivorra/Libros/Libros.htm

Ivorra, C. (2018b). Teorías de conjuntos. Descargado de https://www.uv . es / ivorra/Libros/TCS.pdf

Ortiz, J. R. (1994). El concepto de infinito. Asociación Matemática Venezolana, 1(2).

Paenza, A. (2005). Matemática... ¿estás ahí? Universidad Nacional de Quilmes. Siglo Veintiuno Editores, Colección Ciencia que ladra. Descargado de http://cms.dm.uba.ar/material/paenza/librol/ matematicaEstasAhi.pdf

Rubin, H., y Rubin, J. (1963). Equivalents of the axiom of choice (Vol. 116). Oxford. University Press.

Stein, E., y Shakarchi, R. (2007). Real analysis. Princeton lectures in analysis.

Suppes, P. (1968). Teoría axiomática de conjuntos. Editorial Norma. Cali, Colombia.

Vinogradov, M. (1977). Fundamento de la teoría de los números. MIR, Moscú.Traducción al español. 


\section{Juan Pablo Jorge}

Departamento de Física, Universidad de Buenos Aires, CABA (1428), Argentina; Instituto de Filosofía, Universidad Austral, Pilar (1629), Argentina.

(凶) jorgejpablo@gmail.com

\section{Hernán Luis VÁzQuez}

Departamento de Computación, Universidad de Buenos Aires, CABA (1428), Argentina.

(凹) vazquez.hernan.luis@gmail.com

Recibido: 1 de marzo de 2021.

Aceptado: 14 de julio de 2021.

Publicado en línea: 31 de julio de 2021. 
